\title{
DYNAMICS OF THE RADIX EXPANSION MAP
}

\section{BEN GOERTZEL, HAROLD BOWMAN and RICHARD BAKER}

Department of Mathematics

University of Nevada, Las Vegas

Las Vegas, NV 89154

ABSTRACT The chaotic dynamics of the map $\phi(x)=(\beta x+\alpha)(\bmod 1)$ are studied using Parry's $\beta$ expansion. It is shown that for $1<\beta<2, a \geq 0$, the number of periodic points of period $n$ is $0\left(\beta^{n}\right)$

\section{KEY WORDS AND PHRASES: Dynamics, Chaos, Radix Expansion Map, Invariant Measure} 1993 AMS SUBJECT CLASSIFICATION CODES. 39B20, 26A18

\section{INTRODUCTION}

When $\beta$ is an integer, the dynamics of the $\operatorname{map} \phi(x)=(\beta x+\alpha)(\bmod 1)$ are rather simple. $\phi$ is topologically conjugate to the shift automorphism $\sigma$ on the space $\sum_{\beta}\left\{s_{1} s_{2} \cdots s_{1} \in\{0,1, \cdots, \beta-1\}\right\}$, which, in the terminology of Devaney (1989), implies that it is chaotic (see Def. 2 below). The number of periodic points of period $n$ is $\beta^{n}$, because the period points of $\sigma$ on $\sum_{\beta}$ are precisely the sequences of the form $\overline{s_{1} \cdots s_{n}}, s_{1} \in\{0,1, \cdots, \beta-1\}$. And there is a unique invariant Borel measure, differentiable on $[0,1]$ : the characteristic function $\chi_{[0,1]}($ Parry, 1960).

When $\beta$ is not an integer, things are not quite so tidy. There is still an invariant Borel measure differentiable on $[0,1]$ (Sinai, 1981), although it is zero almost everywhere. However, although the map may still be proved chaotic by demonstrating a conjugacy with a shift map, the space $A_{\beta}$ on which this shift map acts is somewhat problematic. We will deal only with the case $1<\beta<2$, although most of the results generalize straightforwardly. In this case, an analysis of $A_{\beta}$ reveals that the number of periodic points of period $n$ is asymptotically proportional to \#(n), the number of binary sequences $s_{1} s_{2} \cdots s_{n}$ so that:

$$
\begin{aligned}
& \beta^{-1} s_{1}+\cdots+\beta^{-n} s_{n} \leq 1 \\
& \beta^{-1} s_{2}+\cdots+\beta^{-n+1} s_{n} \leq 1 \\
& \quad \vdots \\
& \beta^{-1} s_{n} \leq 1
\end{aligned}
$$

If $X$ is a random variable on $R$, with probability density $f$ so that $\operatorname{supp}(f)=[0,1]$, let $\psi f$ denote the probability density of $\phi(\chi)$. Then, we show that $\psi^{n} \chi_{[0,1]}(0)=\beta^{-n} \#(n)$ It follows that 
$\#(n)=O\left(\beta^{n}\right)$, since $\lim _{n \rightarrow \infty} \psi^{n} \chi_{[0,1]}(0)$ exists by the ergodicity of the mapping $\phi$ (Sinai, 1981) This resul is new and apparently cannot be obtained from standard methods such as kneading theory (Collet and Eckmann, 1980) From here on, we will assume $1<\beta<2, \alpha \geq 0$

\section{INVARIANT MEASURES}

Where $\alpha=0$, Parry (1960) has studied the invariant measure of $\phi$, and shown that it is unique In general, any invariant measure of $\phi$ is characterized by a Frobenius-Perron operator $\psi$.

LEMMA 1 If $0 \leq x \leq 1$, then $\psi f(x)=\beta^{-1}\left[f\left(\beta^{-1} x-\beta^{-1} \alpha\right)+f\left(\beta^{-1} x-\beta^{-1} \alpha+\beta^{-1}\right)+\right.$ $\left.f\left(\beta^{-1} x-\beta^{-1} \alpha+2 \beta^{-1}\right)\right]$

PROOF. $P(\alpha \leq \psi X \leq x)=P\left(\beta^{-1} \alpha-\beta^{-1} \alpha \leq X \leq \beta^{-1} x-\beta^{-1} \alpha\right)+$ $P\left(\beta^{-1} \alpha-\beta^{-1} \alpha+\beta^{-1} \leq X \leq \beta^{-1} x-\beta^{-1} \alpha+\beta^{-1}\right)+P\left(\beta^{-1} \alpha-\beta^{-1} \alpha+2 \beta^{-1} \leq X \leq \beta^{-1} x-\beta^{-1} \alpha+2 \beta^{-1}\right)$

In terms of densities, this means

$$
\int_{\alpha}^{x} \psi f(t) d t=\int_{\beta^{-1} \alpha-\beta^{-1} \alpha}^{\beta^{-1} x-\beta^{-1} \alpha} f(t) d t+\int_{\beta^{-1} \alpha-\beta^{-1} \alpha+\beta^{-1}}^{\beta^{1} x-\beta^{-1} \alpha+\beta^{-1}} f\left(t+\int_{\beta^{-1} \alpha-\beta^{-1} \alpha+2 \beta^{-1}}^{\beta^{-1} x-\beta^{-1} \alpha+2 \beta^{-1}} f(t\right.
$$

Differentiating, one obtains the lemma.

A measure $f$ is invariant under $\phi$ if and only if it is a fixed point of the operator $\psi$ (see Lasota, 1973) From this equation, one may deduce certain simple properties of $\tilde{f}$. For instance, it is easy to see that if $\alpha=0, f(1)=\beta^{-1} f\left(\beta^{-1}\right)=\frac{\beta-1}{\beta} f(0)$.

Next, let us derive a formula for the $n^{\prime}$ th iterate of $\psi$

LEMMA 2. $\psi^{n} f(x)=\beta^{-n} \sum_{i=1}^{2^{n}} f\left(\beta^{-n} x-\beta^{-n} \alpha+\alpha_{1}^{(n)}\right)$, where $\left\{\alpha_{1}^{(n)}, \cdots, \alpha_{2^{n}}^{(n)}\right\}$ is the set of all expansions $\beta^{-1} s_{1}+\beta^{-2} s_{2}+\cdots+\beta^{-n} s_{n}$ so that $s_{1} \in\{0,1\}$ and

$$
\begin{aligned}
& \beta^{-1} x+\beta^{-1} s_{1}+\beta^{-2} s_{2}+\cdots+\beta^{-n} s_{n} \leq 1 \\
& \beta^{-n+1} x+\beta^{-1} s_{2}+\cdots+\beta^{-n+1} s_{n} \leq 1 \\
& \quad \vdots \\
& \beta^{-1} x+\beta^{-1} s_{n} \leq 1
\end{aligned}
$$

PROOF. Lemma 1 takes care of the case $n=1$, so we may proceed by induction. Assume that $a=0$ and that the statement is true for $n=k-1$. Then

$$
\begin{aligned}
& \psi^{k} f(x)=\beta^{-1}\left[\psi^{k-1}\left(\beta^{-1} x\right)+\psi^{k-1}\left(\beta^{-1} x+\beta^{-1}\right)\right]= \\
& \beta^{-1}\left[\beta^{k+1} \sum_{i=1}^{2^{k-1}} f\left(\beta^{k+1} \beta^{-1} x+\alpha_{i}^{(k-1)}\right)+\right. \\
& \left.\beta^{-k+1} \sum_{i=1}^{2^{k-1}} f\left(\beta^{-k+1}\left(\beta^{-1} x+\beta^{-1}\right)+\alpha_{i}^{(k-1)}\right)\right] \\
& =\beta^{-k} \sum_{i=1}^{2^{k-1}}\left[f\left(\beta^{-k} x+\alpha_{i}^{(k-1)}\right)+f\left(\beta^{-k} x+\beta^{-k}+\alpha_{i}^{(k-1)}\right)\right]= \\
& \beta^{k} \sum_{i=1}^{2^{k}} f\left(\beta^{-k} x+\alpha_{i}^{(k)}\right) .
\end{aligned}
$$

This shows that the statement is true for $n=k$ If $\alpha \neq 0$, the lemma follows from the observation that, where $\psi_{1}$ is the operator corresponding to $\phi(x)=(\beta x+\alpha)(\bmod 1)$, and $\psi$ is the operator corresponding to $\phi(x)=\beta x(\bmod 1), \psi_{1} f(x)=\psi f(x-\alpha)$.

This lemma permits us to estimate asymptotically the number $\#(n)$ defined above. The problem of determining $\#(n)$ for arbitrary $n$ is apparently unsolved and seems to be very difficult.

THEOREM $1 \#(n)=O\left(\beta^{n}\right)$

PROOF Take $\alpha=0 \quad \psi^{n} f(\alpha)=\beta^{-n} \sum_{i=1}^{n *} f\left(\alpha_{1}^{(n)}\right) \quad$ Take $f=\chi_{[0.1]}$, then $\psi^{n} f(\alpha)=\beta^{-n} \#(n) \quad \psi^{n}$ converges to an invariant measure, because $\psi$ is ergodic (Sinai, 1981) Thus $\#(n)=O\left(\beta^{n}\right)$ 


\section{APPROXIMATION WITH INTERVAL MAPS}

Computer simulations have played a large role in the development of the theory of chaotic dynamical systems. One way to simulate the probabilistic behavior of a chaotic map like $\phi$ is to approximate the map by a sequence of interval maps.

Given a measure $f$ so that $\operatorname{supp}(f)=[0,1]$, let $P_{f}^{(n)}=P^{(n)}=\left(P_{1}^{(n)}, \cdots, P_{n}^{(n)}\right)^{T}$, where $P_{1}=\int_{(1-1) / n}^{1 / n} f(x) d x$ Let $\psi_{n}$ be the $n \times n$ matrix defined by $\left(\psi_{n} P^{(n)}\right)_{1}=\int_{\frac{1-1}{n}}^{1 / n} \psi f(x) d x$

LEMMA 3. $\exists k>0$ so that $\left(\psi_{n}^{k}\right)_{l j}>0$ for all $i, j$.

PROOF. $\left(\psi_{n}^{k}\right)_{l j}$ is the probability that, after $k$ iterations of $\phi$, the image of a point selected from a uniform distribution on $\left[\frac{l-1}{n}, \frac{J}{n}\right)$ is in $\left[\frac{l-1}{n}, \frac{l}{n}\right)$ Because $\phi$ is ergodic, for any two open intervals $U$ and $V$ there is some $k$ so that the Lebesgue measure of $\psi(U) \cap V$ is nonzero

THEOREM $2 \psi_{n}$ has a unique fixed point $\tilde{P}^{(n)}$

PROOF Since $\left\|\psi_{n}\right\|_{1}=1, P\left(\psi_{n}\right) \leq 1$. And since $\sum_{j=1}^{n}\left(A_{n}\right)_{l j}=1,1$ is an eigenvalue of $A_{n}^{T}$ with corresponding eigenvector $(1, \cdots, 1)$, and hence 1 is an eigenvalue of $A_{n}$ and $\rho\left(A_{n}\right)=1$. According to a standard linear algebra result, Lemma 3 implies that the multiplicity of 1 is 1 Thus there is a unique eigenvector $\tilde{P}^{(n)}$ corresponding to the eigenvalue 1 .

Given this result, it is easy to see that these $\tilde{P}^{(n)}$ converge to the invariant measure. For any $x \in[0,1]$, let $I_{n}(x)$ denote the interval $\left[\frac{i-1}{n}, \frac{i}{n}\right)$ which contains $x$. Let $S^{n}$ denote the set of all step functions constant on each interval $\left[\frac{i-1}{n}, \frac{t}{n}\right)$. Let $\tilde{f}^{(n)}$ be the element of $S^{n}$ naturally induced by $\tilde{P}^{(n)}$ Then we have

THEOREM 3. $\lim _{l \rightarrow \infty} \tilde{f}^{(n)}=\tilde{f}$.

These results show that the interval maps $\psi_{n}$ are qualitatively faithful representations of $\psi$. The chaotic behavior of $\phi$ is necessarily absent from any discrete approximation, but the probabilistic implications of this chaos are accurately mirrored.

4. DYNAMICS

DEFINITION 1. Where $s=s_{1} \cdots s_{n} \cdots$ is a binary sequence, let $B_{\beta}(s)=\beta^{-1} s_{1}+\beta^{-2} s_{2}+\cdots \beta^{-n} s_{n}+\cdots \quad$ Let $F_{\beta}$ be the set of all $s=s_{1} \cdots s_{n}$ or $s=s_{1} s_{2} \cdots$ so that $B_{\beta}(s) \geq 1$ Let $A_{\beta}$ be the set of all $s=s_{1} s_{2} \cdots$ so that $s_{k} s_{k+1} \cdots s_{k+m} \notin F_{\beta}, s_{k} s_{k+1} \cdots \notin F_{\beta}$, for any $k$ and $\phi$.

$F_{\beta}$ is the set of all "forbidden subsequences", and $A_{\beta}$ is the set of all sequences containing no forbidden subsequences.

LEMMA 4. For each $x \in[0,1]$ there is a unique binary sequence $s \in A_{\beta}$ so that $B_{\beta}(s)=x$.

PROOF Existence is clear, one forms an expansion $B_{\beta}$ exactly as one forms an expansion in an integer base. To show uniqueness, assume $x=B_{\beta}\left(s_{1} s_{2} \cdots s_{n-1} l s_{n+1} \cdots\right)$ and $x=B_{\beta}\left(s_{1} s_{2} \cdots s_{n-1} 0 s_{n+1}^{\prime} s_{n+2}^{\prime} \cdots\right)$ Then the second expansion is not in $A_{\beta}$

Chaos may be defined in many different ways Here we will adopt the topological approach found in Devaney (1989)

DEFINITION 2 A function $r[0,1] \rightarrow[0,1]$ is chaotic if i) it is topologically transitive,

ii) its periodic points are dense, iii) it is sensitive to initial conditions

LEMMA 5. $\phi[0,1] \rightarrow[0,1]$ is topologically conjugate to the shift map $\sigma A_{\beta} \rightarrow A_{\beta}$, where $\sigma\left(s_{1} s_{2} s_{3} \cdots\right)=s_{2} s_{3} \cdots$

PROOF. Follows from Lemma 4 by standard arguments 
THEOREM $4 \phi$ is chaotic on $[0,1] \rightarrow[0,1]$

PROOF. i) $\phi$ is topologically transitive because, where $\left\{w_{1} w_{2} w_{3}, \cdots\right\}$ is an enumeration of $A_{\beta}, s=w_{1} 00 w_{2} 00 w_{3} 00 \cdots$ is in $A_{\beta}$ and $B_{\beta}(s)$ is a dense orbit. ii) The set of periodic points in $\phi$ of period $n$ is $\left\{B_{\beta}(s) . s \in A_{\beta}, s=s_{1}, \cdots s_{n}, s_{1} \in\{0,1\}\right\}$ For example, everything of the form $B_{\beta}\left(\overline{s_{1} s_{2} \cdots s_{n} 00}\right)$ is periodic, and these points are clearly dense. iii) Follows from standard arguments

THEOREM 5 If $a$ and $\beta$ are rational, all periodic points of $\phi$ are rational.

PROOF. if $a=0$, a little algebra shows that all periodic points are of the form $\left[s_{1} \beta^{-1}+\cdots+s_{n} \beta^{n}\right] /\left[\beta^{n}-1\right], s_{l} \in\{0,1\}$. The general case follows similarly

These results can also be obtained by more conventional methods (Collet and Eckmann, 1980). However the present techniques tell us considerably more about the periodic points of $\phi$ than Theorem 4 requires. Let $\operatorname{Per}_{n}(\phi)$ denote the set of periodic points of $\phi$ of period $n$. For $\alpha=0$ and $\beta$ integral, it is obvious that $\operatorname{Card}\left[\operatorname{Per}_{n}(\phi)\right]=\beta^{n}$. This result cannot be directly generalized to the case of nonintegral $\beta$, since $\operatorname{Card}\left[\operatorname{Per}_{n}(\phi)\right]$ is integral but $\beta^{n}$ is not. However, the following result is an asymptotic generalization.

THEOREM 6. $\operatorname{Per}_{n}(\phi)=O\left(\beta^{n}\right)$

PROOF: $\left\{B_{\beta}(s): s \in A_{\beta}, s=\overline{s_{1} \cdots s_{n-2}}, s,\{0,1\}\right\} \subset \operatorname{Per}_{n}(\phi)$, but $\operatorname{Per}_{n}(\phi) \subset\left\{B_{\beta}(s): s \in A_{\beta}, s=\overline{s_{1} \cdots s_{n}}, s_{1} \in\{0,1\}\right\}$. So $\#(n-2) \leq \operatorname{Card}\left[\operatorname{Per}_{n}(\phi)\right] \leq \#(n)$, and the result follows from Theorem 1.

NOTE: Since submitting this paper, the authors have become aware of unpublished work by Leo Flatto which contains the result $\operatorname{Card}\left[\operatorname{Per}_{n}(\phi)\right]=O\left(\beta^{n}\right)$ for the case $\alpha=0$. Flatto's techniques are combinatorial rather than operator-theoretic, and apparently cannot be extended to the case $\alpha \neq 0$. We would like to thank J. Lagarias for informing us of Flatto's work.

\section{REFERENCES}

1. BLANCHARD, F. (1989), ß-Expansions and Symbolic Dynamics, Theoretical Computer Science $65,131-141$.

2. COLLET, P. and ECKMANN, J.P (1980): Iterated Maps of the Interval as Dynamical Systems, Birkhauser, Boston, Massachusetts.

3. DEVANEY, R.L. (1989): Chaotic Dynamical Systems, Addison-Wesley, New York, New York.

4. GRABNER, P. and TICHEY, R. (1991): Manuscripta Mathematica 70, 311-324.

5. KIRCHGRABER, U. and STOFFER, D. (1989), Z. Angew. Math. Mech 69, 175-185.

6. LASOTA, A. (1973), Aequationes Math 9, 193-200.

7. LASOTA, A. and YORKE, J (1982), Trans. A.M.S. 273, 375-384.

8. PARRY, W. (1960), Acta. Math. Acad. Sci. Hung. 10, 401-416

9 RECHARD, O.W. (1956), Duke Math. J 23, 477-488

10. RENYI, A (1957), Acta, Math. Acad. Sci. Hung 7, 477-493

11 SINAI, J.G. and FOMIN, S.V (1981), Introduction to Ergodic Theory, Princeton University Press, Princeton, New Jersey. 


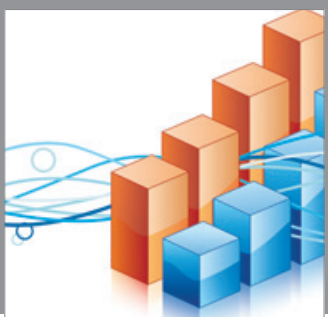

Advances in

Operations Research

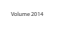

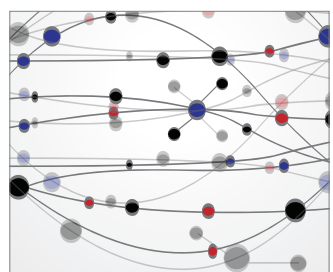

\section{The Scientific} World Journal
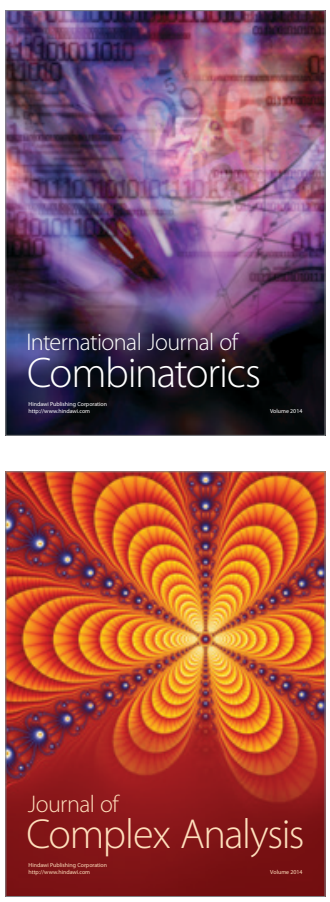

International Journal of

Mathematics and

Mathematical

Sciences
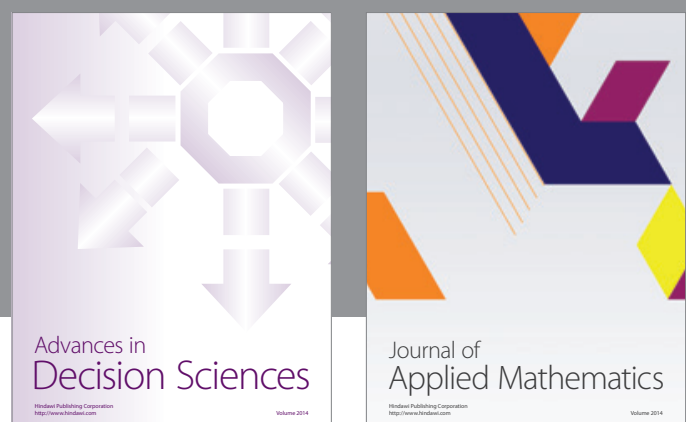

Journal of

Applied Mathematics
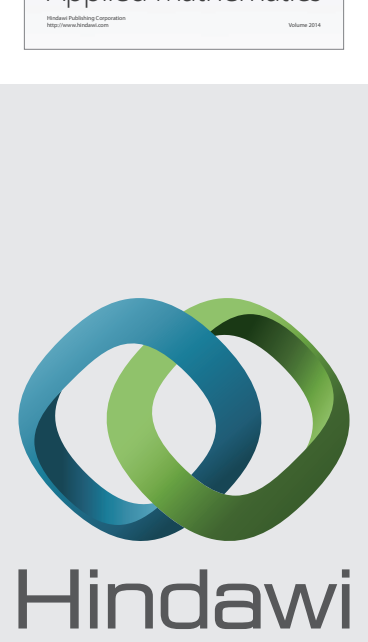

Submit your manuscripts at http://www.hindawi.com
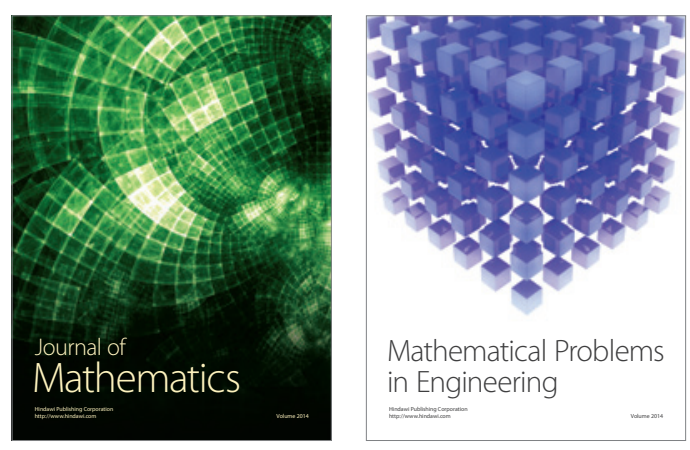

Mathematical Problems in Engineering
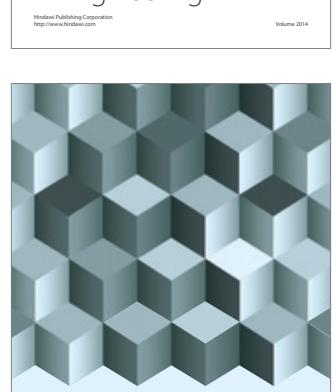

Journal of

Function Spaces
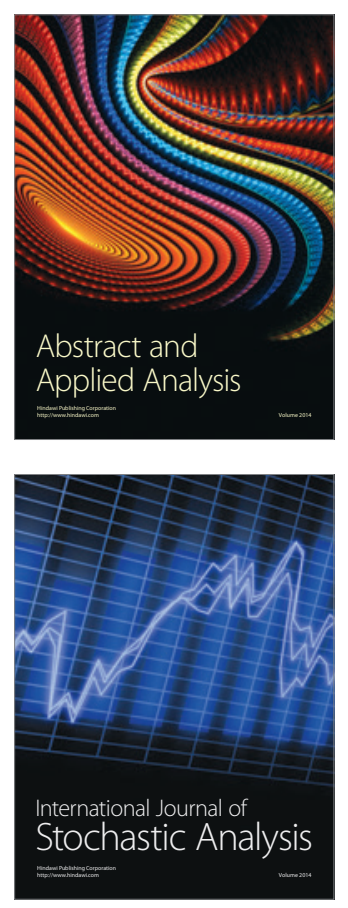

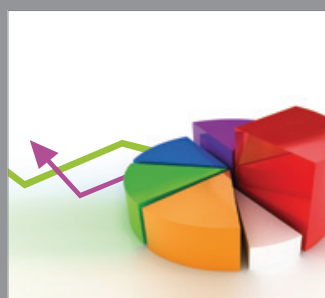

ournal of

Probability and Statistics

Promensencen
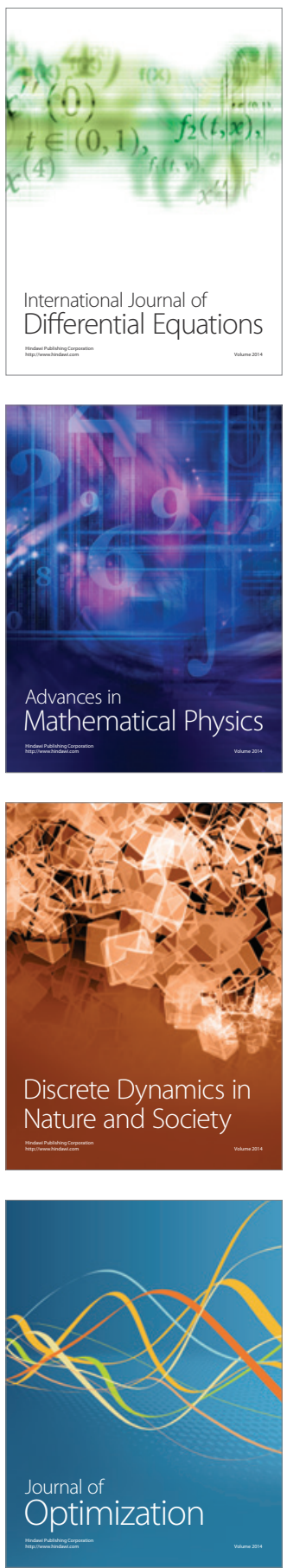